\title{
Immediate and short-term effects of $35 \%$ tension Kinesio taping on handgrip strength in healthy females
}

\author{
DOI: https://doi.org/10.5114/pq.2021.105746
}

\author{
Waleed S. Mahmoud ${ }^{1,2}$, Ehab Mohamed Kamel ${ }^{3,4}$, Mohamed Raafat Atteya ${ }^{4,5}$, Ahmed Abdelmoniem Ibrahim ${ }^{5,6}$, \\ Saud M. Alrawaili', Hisham Mohamed Hussein ${ }^{2,5}$ (1) \\ ${ }^{1}$ Department of Physical Therapy and Health Rehabilitation, College of Applied Medical Sciences, Prince Sattam \\ bin Abdulaziz University, Riyadh, Alkharj, Saudi Arabia \\ ${ }^{2}$ Department of Basic Science, Faculty of Physical Therapy, Cairo University, Giza, Egypt \\ ${ }^{3}$ College of Public Health and Health Informatics, University of Hail, Ha'il, Saudi Arabia \\ ${ }^{4}$ Department of Physical Therapy, El Helal Hospital, Cairo, Egypt \\ ${ }^{5}$ Department of Physical Therapy, College of Applied Medical Sciences, University of Hail, Ha'il, Saudi Arabia \\ ${ }^{6}$ Department of Physical Therapy, Cairo University, Giza, Egypt
}

\section{Abstract}

Introduction. Kinesio taping is one of the commonly used treatment modalities in rehabilitation. When applied appropriately, it may improve muscle strength and performance. This study aimed to determine the immediate and short-term effect of $35 \%$ tension $\mathrm{Ki}$ nesio taping on handgrip strength in healthy females.

Methods. Overall, 60 healthy female subjects aged 19-30 years were randomly assigned to 2 groups: the tension Kinesio taping group, who received taping with $35 \%$ tension, and the control group, who received the same taping technique but with no tension. A hand-held dynamometer was used to measure handgrip strength in all participants. The measurements were taken before taping, as well as at 30 minutes and 24 and 48 hours of taping.

Results. Handgrip strength significantly improved in both groups after taping $(p<0.05)$. Comparing with the control group, the tension Kinesio taping group demonstrated more significant results at 30 minutes and 24 and 48 hours $(p<0.05)$.

Conclusions. Taping with $35 \%$ tension produces an immediate and short-term increase in handgrip strength in healthy females. Key words: athletic tape, hand strength, healthy volunteers, females

\section{Introduction}

Kinesio taping (KT) has become one of the commonly used treatment modalities in the recent years. This treatment method is relatively new: Kenzo Kase first created it in 1973 [1]. Owing to its flexibility, the use of KT has no adverse effects on joint range of motion. The adhesive surface of KT is characterized by a wave-like grain design, which provides a considerable amount of elasticity [2].

Recently, KT has been used in a variety of fields with different goals: alleviating pain [3], augmenting joint range of motion [4], correcting fascial mechanics [5], stimulating the sensory system, improving circulation [6], and enhancing muscle performance [7].

The strength of the handgrip muscles is one of the key factors determining muscle performance, which must be assured during any rehabilitation program for the upper extremities [8]. Loss of strength prevents the subject from performing necessary daily activities and occupational tasks [9, 10].

Improving handgrip strength via a simple, applicable, and easy method such as KT is a significant concern in rehabilitation [11]. It can lead to recovery in musculoskeletal injuries and neuromuscular re-education [12, 13]. KT has gained acceptance among rehabilitation and sports professionals as an effective technique for different conditions [14].

The literature shows a lack of consistency regarding the amount of tension required to produce the appropriate handgrip strength. To enhance muscle power, Kenzo Kase sug- gested taping with 25-35\% tension [15]. However, other researchers recommended $50 \%$ tension, while Atrib Zanchet et al. [16] demonstrated that KT with $25 \%$ tension did not demonstrate any changes in the handgrip strength.

Limmer et al. [17] reported that KT over the wrist flexors and extensors might not improve muscle strength; however, it could prevent muscle fatigue during muscle endurance performance tasks in tennis players.

In terms of timing, a previous study stated that the most significant effect of taping as measured by percent changes in handgrip power appeared after 30 minutes in men and 90 minutes in women [7]. Another study compared different durations of KT on the handgrip and pinch grip strengths: the evaluations were performed immediately, as well as 24 and 48 hours after the tape application. The results revealed that the desired effects occurred after 48 hours of application [18]. Another study evaluated knee extensor torque after 24 hours of KT application, demonstrating that 1 day of KT application was insufficient to induce a measurable change in quadriceps strength [19].

In light of the previously described controversies - and the popularity of using KT in the rehabilitation of hand injuries - well designed studies on the efficacy of different tensions applied with taping techniques are needed. Thus, this study aimed to investigate (1) the effect of KT with $35 \%$ tension, (2) the influence of KT duration on handgrip strength in healthy subjects.

Correspondence address: Hisham M. Hussein, Physiotherapy Department, College of Applied Medical Sciences, University of Hail, P.O. Box: 2240, Ha'il, Saudi Arabia, e-mail: drhisham3000@cu.edu.eg, https://orcid.org/0000-0003-2184-6147 


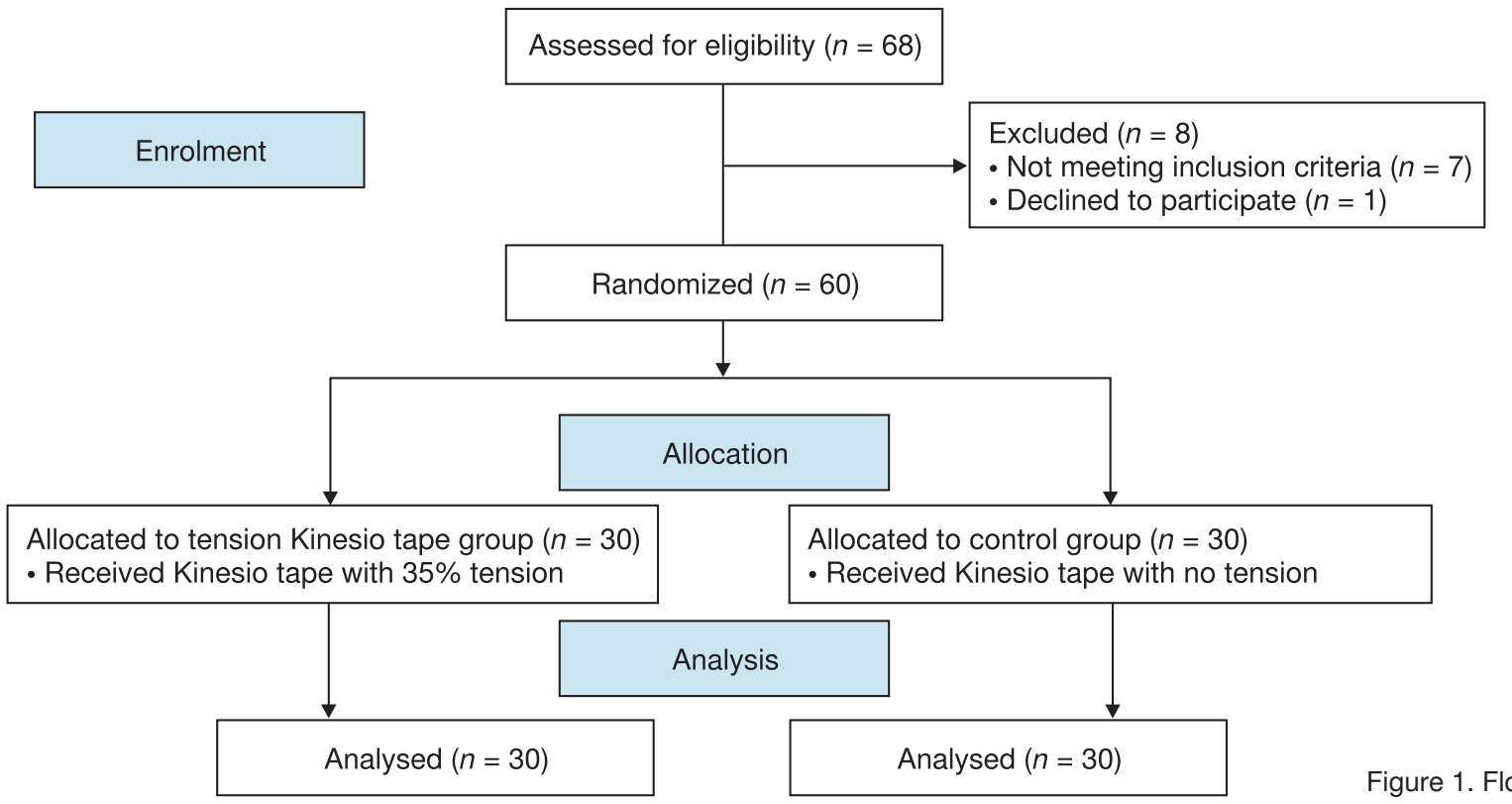

\section{Subjects and methods}

\section{Design}

This randomized double-blind controlled clinical trial was conducted between January and June 2019.

\section{Participants}

Overall, 60 healthy female participants were recruited from the local community with verbal and written announcement. Included were healthy females aged 19-30 years. Subjects were excluded if they (1) had a localized or systemic disease or injury affecting the function of the upper extremities, (2) were athletes, and (3) received analgesic or muscle relaxant medications (Figure 1 ).

\section{Randomization}

The participants received clear instructions about the purposes and procedures of the study, as well as detailed explanation of their rights. They were allocated into 2 equal groups with the use of permuted blocks. The randomization was performed by the third author, who was not involved in either the assessment or treatment.

\section{Intervention}

The first group, the tension Kinesio taping (tKT) group, received $\mathrm{KT}$ with $35 \%$ tension over the extensor surface of the forearm. The second group was a control group and received the same taping technique but with no tension.

An experienced therapist (the fourth author) applied the tape to the participants in both groups. The tKT group subjects received I-shaped taping, in accordance with the guidelines stated by Mohammadi et al. [7] and adopted recently by Kim and Kim [1]. The extensor surface of the dominant forearm was cleaned with an alcohol cotton swap before applying the tape. The therapist instructed the participants to sit on a chair with a straight back, assuming the upright position and resting her feet on the ground. The examined arm was held against the body, with the elbow joint kept at $90^{\circ}$, the forearm pronated, and the wrist joint in the neutral position.

For the tKT group, the length of the tape was determined as the distance between the point located $2 \mathrm{~cm}$ distally to the lateral epicondyle of the humerus and the radial styloid process. The tape ends (anchors) were $4 \mathrm{~cm}$ (2 in each end). The $35 \%$ tension was calculated with the following formula:

$$
(L-4 / 1.35)+4
$$

where $L$ is the tape length, 4 represents the length of the anchors, and 1.35 depicts the required tension [20].

The control group received taping with no tension, applied between the previously mentioned points (Figure 2). The taping was administered from proximal to distal. The participants were instructed to leave the tape in place for the entire period of the study (48 hours).

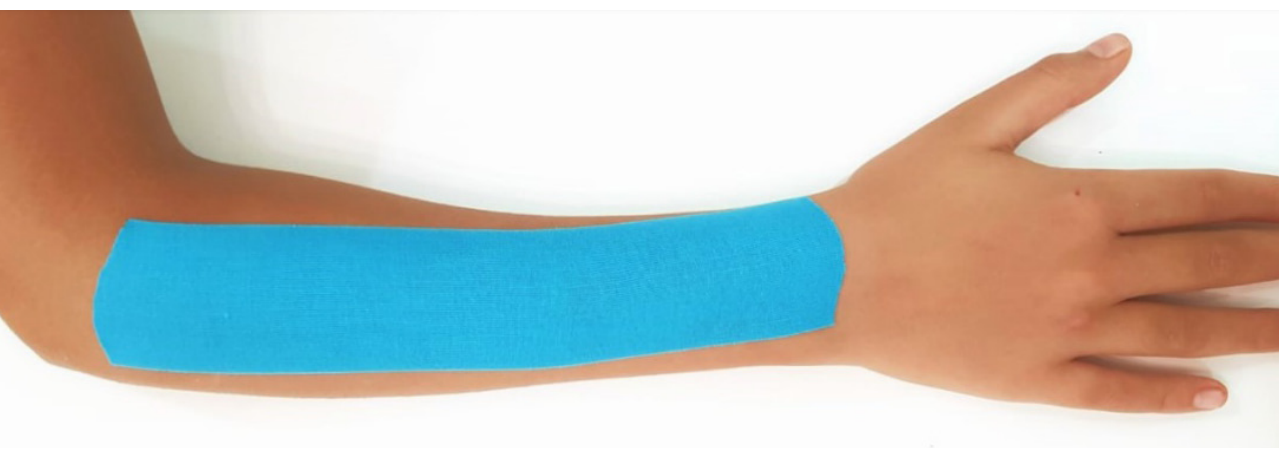
taping on the dorsal surface of forearm 


\section{Outcome measure}

The assessor (the second author) measured the strength of the handgrip using a recently calibrated Lafayette push hand-held dynamometer (model 01163, Lafayette Instrument Co., Lafayette, IN, USA). The dynamometer has a dual pointer system to retain the maximum effort. The testing range is on a dual scale of $0-100 \mathrm{kG}$. The device is a valid and reliable tool for assessing isometric hand muscle strength [21].

The participants were instructed to sit on a chair with armrests. The shoulder was slightly abducted, the elbow was at a right angle, and the forearm was in the mid-supination/pronation position. The subjects were asked to squeeze the handle of the dynamometer using maximum effort for $3 \mathrm{sec}-$ onds and then relax for 1 minute. This measurement was repeated 3 times, and the average of the 3 readings was considered the baseline record for maximum grip strength. The testing procedure was performed with the dominant hand before taping and repeated after 30 minutes and 24 and 48 hours of taping.

\section{Sample size calculation}

The sample size was estimated on the basis of the expected effect size $(d=0.3)$ with $80 \%$ power and a probability of 0.05 . The analysis indicated a total sample size of 58 participants (29 per group); however, we recruited 60 participants in anticipation of dropout.

\section{Statistical analysis}

The statistical analyses used the IBM Statistical Package for the Social Sciences (SPSS) software, version 23 (Chicago, USA). All data were expressed as mean \pm standard deviation. They were normally distributed, which was verified with the Shapiro-Wilk test. An unpaired $t$-test was applied to determine any changes between the 2 groups regarding the demographic characteristics and handgrip strength. Repeated measure analysis of variance (ANOVA) evaluated within-subject changes in handgrip strength at different measurement time points in both groups. The Bonferroni post-hoc test implied where changes occurred across different time measurements. Statistical significance was defined as $5 \%$.

\section{Ethical approval}

The research related to human use has complied with all the relevant national regulations and institutional policies, has followed the tenets of the Declaration of Helsinki, and has been approved by the ethical committee of College of Applied Medical Sciences, the University of Hail (No. PT:47/2020).

\section{Informed consent}

Informed consent has been obtained from all individuals included in this study.

\section{Results}

There were no significant differences in terms of age, height, weight, or body mass index between the 2 groups (Table 1). The comparison of handgrip strength scores between the 2 groups revealed that the IKT group presented significantly improved handgrip strength after 30 minutes and 24 and 48 hours $(p=0.02, p=0.000$, and $p=0.000$, respectively) vs. the control group (Table 2 ).

The within-subject results in the tKT group showed a significant increase in handgrip strength compared with baseline (Wilks' lambda $=0.11, \mathrm{~F}(3,27)=74.13, p<0.05$, $\eta^{2}=0.89$ ), as illustrated in Table 2. However, the Bonferroni post-hoc test revealed no significant improvements in handgrip strength when comparing 24 with 48 hours of taping $(p=1)$ (Figure 3).

Regarding the control group, taping without tension demonstrated a significant increase in the handgrip strength when compared with baseline (Wilks' lambda $=0.39, \mathrm{~F}(3,27)=$ 13.96, $\left.p<0.05, \eta^{2}=0.60\right)$. This improvement was evident at 30 minutes and 24 and 48 hours of taping. However, the Bonferroni post-hoc test reported no significant differences in handgrip strength between 30 minutes and 24 and 48 hours of taping $(p>0.05)$.

Table 1. Demographic characteristics of participants

\begin{tabular}{|l|c|c|c|}
\hline Variables & $\begin{array}{c}\text { Tension Kinesio taping group }(n=30) \\
(\text { mean } \pm S D)\end{array}$ & $\begin{array}{c}\text { Control group }(n=30) \\
(\text { mean } \pm S D)\end{array}$ & $p$ \\
\hline Age (years) & $25.9 \pm 3.34$ & $26.46 \pm 2.16$ & 0.44 \\
\hline Weight $(\mathrm{kg})$ & $59.26 \pm 9.86$ & $55.8 \pm 8.05$ & 0.518 \\
\hline Height $(\mathrm{cm})$ & $157.8 \pm 5.50$ & $159.3 \pm 3.98$ & 0.240 \\
\hline Body mass index & $23.87 \pm 4.40$ & $21.97 \pm 3.17$ & 0.161 \\
\hline
\end{tabular}

Table 2. Changes in handgrip strength $(\mathrm{kG})$ between groups at baseline and after 30 minutes and 24 and 48 hours

\begin{tabular}{|l|c|c|c|}
\hline Time of measurement & $\begin{array}{c}\text { Tension Kinesio taping group }(n=30) \\
(\text { mean } \pm S D)\end{array}$ & $\begin{array}{c}\text { Control group }(n=30) \\
(\text { mean } \pm S D)\end{array}$ & $p$ \\
\hline Baseline & $16.96 \pm 4.19$ & $16.13 \pm 4.19$ & 0.45 \\
\hline After 30 minutes & $19.36 \pm 3.96^{*}$ & $16.93 \pm 3.93^{*}$ & 0.02 \\
\hline After 24 hours & $21.9 \pm 3.66^{*}$ & $17.36 \pm 3.63^{*}$ & 0.000 \\
\hline After 48 hours & $22.1 \pm 3.78^{*}$ & $17.23 \pm 3.55^{*}$ & 0.000 \\
\hline
\end{tabular}

\footnotetext{
* significant when compared with baseline
} 


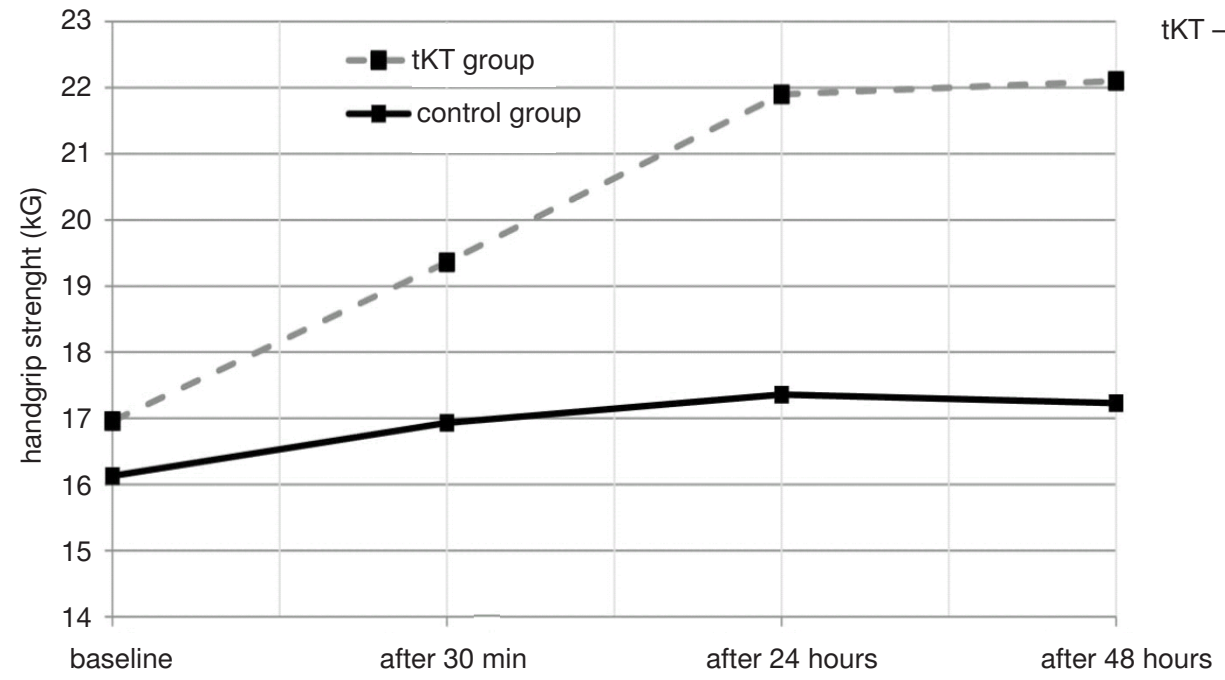

Figure 3. Influence of Kinesio taping application time on handgrip strength in the 2 groups

\section{Discussion}

The purpose of this study was to assess the immediate and short-term effect of KT with $35 \%$ tension on handgrip strength. The results demonstrated a significant increase in the strength of the handgrip when $35 \%$ tension was used; this significant effect was observed at 30 minutes and 24 and 48 hours of taping.

Regarding the tKT group, the handgrip strength reached its peak value at 24 hours and then plateaued from 24 to 48 hours. Meanwhile, the handgrip strength in the control group reached the peak at 24 hours, then started to decline, and reached borderline statistical significance at 48 hours of taping $(p=0.047)$.

The current results agree with those demonstrated by Vilela Lemos et al. [15]. These authors concluded that taping with $35 \%$ tension could effectively increase the handgrip strength in normal subjects.

In contrast to our findings, Merino-Marban et al. [22] reported no changes in handgrip strength after using 30\% tension taping. In that study, the tape was applied to the flexor surface of the forearm.

In other research, taping with $50 \%$ tension demonstrated favourable results $[1,7]$, with an immediate improvement in a study conducted by Kim and Kim [1]. Unfortunately, the sample in that study was small and the gender was not specified. Mohammadi et al. [7] reported similar results after $50 \%$ tension taping. Moreover, they noticed that the onset of the taping effects was relatively slower in women than men. In the current study, both groups reached peak grip strength after 24 hours of taping.

Taping pulls the skin upward when applied with tension, and thus more space is provided for contraction by lifting the soft tissues [23]. Additionally, the connections that may exist between the skin and proprioceptors might increase the number of motor unit recruitments after cutaneous stimulation by the tension of taping [15]. This recruitment hypothesis is supported by the increased electrical activity inside the muscle observed after taping the related cutaneous tissue [24-26].

Taping without tension can evoke a placebo effect and produce changes in muscle strength [27]. This effect was short-lasting as it declined after 24 hours of taping, as shown in the current study. Another explanation for the improvement reported in the control group is that KT produces cuta- tivity and increase the strength of the handgrip even without tension.

\section{Limitations}

The current study has 2 limitations: it did not consider the difference in taping response that may be found between males and females, and it did not investigate the intermediate effect. Consequently, future studies should take into account both gender differences and the intermediate effect of taping

\section{Conclusions}

Both KT with $35 \%$ tension and with no tension can produce an immediate and short-term increase in handgrip strength. However, 35\% tension taping resulted in higher strength that persisted for a longer time.

\section{Disclosure statement}

No author has any financial interest or received any financial benefit from this research.

\section{Conflict of interest}

The authors state no conflict of interest.

\section{References}

1. Kim JY, Kim SY. Effects of Kinesio tape compared with non-elastic tape on hand grip strength. J Phys Ther Sci. 2016;28(5):1565-1568; doi: 10.1589/jpts.28.1565.

2. Nakajima MA, Baldridge $C$. The effect of Kinesio ${ }^{\circledR}$ tape on vertical jump and dynamic postural control. Int J Sports Phys Ther. 2013;8(4):393-406.

3. Nemitalla Added MA, Oliveira Pena Costa L, Fukuda TY, Galace de Freitas D, Salomão EC, Monteiro RL, et al. Efficacy of adding the Kinesio taping method to guideline-endorsed conventional physiotherapy in patients with chronic nonspecific low back pain: a randomised controlled trial. BMC Musculoskelet Disord. 2013;14(1): 301; doi: 10.1186/1471-2474-14-301.

4. Wilson V, Douris P, Fukuroku T, Kuzniewski M, Dias J, Figueiredo $P$. The immediate and long-term effects of Kinesiotape ${ }^{\circledR}$ on balance and functional performance. Int J Sports Phys Ther. 2016;11(2):247-253.

5. Tu SJ, Woledge RC, Morrissey D. Does 'Kinesio tape' alter thoracolumbar fascia movement during lumbar flex- 
ion? An observational laboratory study. J Bodyw Mov Ther. 2016;20(4):898-905; doi: 10.1016/j.jbmt.2016.04. 007.

6. Malicka I, Rosseger A, Hanuszkiewicz J, Woźniewski M. Kinesiology taping reduces lymphedema of the upper extremity in women after breast cancer treatment: a pilot study. Prz Menopauzalny. 2014;13(4):221-226; doi: 10.5114/pm.2014.44997.

7. Mohammadi HK, Kalantari KK, Naeimi SS, Pouretezad M, Shokri E, Tafazoli M, et al. Immediate and delayed effects of forearm Kinesio taping on grip strength. Iran Red Crescent Med J. 2014;16(8):e19797; doi: 10.5812/ircmj.19797.

8. Alqahtani B, Alenazi A, Alshehri M, Alqahtani M, Elnaggar R. Reference values and associated factors of hand grip strength in elderly Saudi population: a cross-sectional study. BMC Geriatr. 2019;19(1):271; doi: 10.1186/ s12877-019-1288-7.

9. Kjeken I, Smedslund G, Moe RH, Slatkowsky-Christensen $B$, Uhlig T, Hagen KB. Systematic review of design and effects of splints and exercise programs in hand osteoarthritis. Arthritis Care Res. 2011;63(6):834-848; doi: 10.1002/acr.20427.

10. Mitsukane M, Sekiya N, Himei S, Oyama K. Immediate effects of repetitive wrist extension on grip strength in patients with distal radial fracture. Arch Phys Med Rehabil. 2015;96(5):862-868; doi: 10.1016/j.apmr.2014.09.024.

11. Ahn J-Y, Han J-S, Min K-S. Experimental study for defining range of motion required for daily activities in wrist joint. J Korean Orthop Assoc. 2000;35(5):797-802; doi: 10.4055/jkoa.2000.35.5.797.

12. Ozmen T, Aydogmus M, Dogan H, Acar D, Zoroglu T, Willems M. The effect of Kinesio taping on muscle pain, sprint performance, and flexibility in recovery from squat exercise in young adult women. J Sport Rehabil. 2016; 25(1):7-12; doi: 10.1123/jsr.2014-0243.

13. Horasart A, Klomjai W, Bovonsunthonchai S. Immediate effect of Kinesio tape on gait symmetry in patients with stroke: a preliminary study. Hum Mov. 2020;21(1):73-81; doi: 10.5114/hm.2020.88156.

14. Krause F, Dust K, Banzer W, Vogt L. Cohort survey on prevalence and subjectively-perceived effects of Kinesiotape. Dtsch Z Sportmed. 2017;68:116-120; doi: 10.5960/dzsm.2017.272.

15. Vilela Lemos T, Pereira KC, Protássio CC, Barbosa Lucas L, Matheus JPC. The effect of Kinesio taping on handgrip strength. J Phys Ther Sci. 2015;27(3):567-570; doi: 10.1589/jpts.27.567.

16. Atrib Zanchet M, Chirivino Antunes da Rocha AC, Boscolo del Vecchio F. Effects of Kinesiotaping on handgrip isometric strength in athletes. Int Phys Med Rehab J. 2017;2(1):184-188; doi: 10.15406/ipmrj.2017.02.00041.

17. Limmer M, Buck S, de Marées M, Roth R. Acute effects of Kinesio taping on muscular strength and endurance parameters of the finger flexors in sport climbing: a randomised, controlled crossover trial. Eur J Sport Sci. 2020; 20(4):427-436; doi: 10.1080/17461391.2019.1633415.

18. Murugan S, Shruti $P$, Tejal $P$, Charul $P$. Facilitatory effects of Kinesio taping on forearm extensors in improving hand grip and pinch grip strength. Sch Acad J Biosci. 2016; 4(6):478-482; doi: 10.21276/sajb.2016.4.6.5.

19. Dos Santos Soares M, Lopes AJ, dos Santos Vigario P, Pugila Souza M, Figueiredo da Costa H, Felicio LR. Does the Kinesio tape provide more knee extensor torque? Asian J Sports Med. 2018;9(1):e63946; doi: 10.5812/ asjsm.63946.
20. Shakeri S, Kalantari KK, Baghban AA. The effect of tension and the extent of coverage of Kinesio tape on the knee extensor torque in healthy young people. World J Phys Rehabil Med. 2019;3(1):1009.

21. Hébert LJ, Maltais DB, Lepage C, Saulnier J, Crête M, Perron M. Isometric muscle strength in youth assessed by hand-held dynamometry: a feasibility, reliability, and validity study. Pediatr Phys Ther. 2011;23(3):289-299; doi: 10.1097/PEP.0b013e318227ccff.

22. Merino-Marban R, Mayorga-Vega D, Fernandez-Rodriguez E. Acute and $48 \mathrm{~h}$ effect of Kinesiotaping on the handgrip strength among university students. J Hum Sport Exerc. 2012;7(4):741-747; doi: 10.4100/jhse.2012. 74.02 .

23. Chang H-Y, Chou K-Y, Lin J-J, Lin C-F, Wang C-H. Immediate effect of forearm Kinesio taping on maximal grip strength and force sense in healthy collegiate athletes. Phys Ther Sport. 2010;11(4):122-127; doi: 10.1016/j. ptsp.2010.06.007.

24. Chen S-M, Alexander R, Lo SK, Cook J. Effects of functional fascial taping on pain and function in patients with non-specific low back pain: a pilot randomized controlled trial. Clin Rehabil. 2012;26(10):924-933; doi: 10.1177/ 0269215512441484.

25. Hsu Y-H, Chen W-Y, Lin H-C, Wang WTJ, Shih Y-F. The effects of taping on scapular kinematics and muscle performance in baseball players with shoulder impingement syndrome. J Electromyogr Kinesiol. 2009;19(6): 1092-1099; doi: 10.1016/j.jelekin.2008.11.003.

26. Słupik A, Dwornik M, Białoszewski D, Zych E. Effect of Kinesio taping on bioelectrical activity of vastus medialis muscle. Preliminary report. Ortop Traumatol Rehabil. 2007;9(6):644-651.

27. Mak DN-T, Au IP-H, Chan M, Chan ZY-S, An WW, Zhang JH, et al. Placebo effect of facilitatory Kinesio tape on muscle activity and muscle strength. Physiother Theory Pract. 2019;35(2):157-162; doi: 10.1080/09593985. 2018.1441936. 